\title{
Single-particle versus pair superfluidity in a bilayer system of dipolar bosons
}

\author{
A. Macia, ${ }^{1}$ G. E. Astrakharchik, ${ }^{1}$ F. Mazzanti, ${ }^{1}$ S. Giorgini, ${ }^{2}$ and J. Boronat ${ }^{1}$ \\ ${ }^{1}$ Departament de Física i Enginyeria Nuclear, Universitat Politècnica de Catalunya, Campus Nord B4-B5, E-08034 Barcelona, Spain \\ ${ }^{2}$ Dipartimento di Fisica, Università di Trento and INO-CNR BEC Center, I-38123 Povo, Trento, Italy
}

(Received 24 July 2014; published 21 October 2014)

\begin{abstract}
We consider the ground state of a bilayer system of dipolar bosons, where dipoles are oriented by an external field in the direction perpendicular to the parallel planes. Quantum Monte Carlo methods are used to calculate the ground-state energy, the one-body and two-body density matrix, and the superfluid response as a function of the separation between layers. We find that by decreasing the interlayer distance for fixed value of the strength of the dipolar interaction, the system undergoes a quantum phase transition from a single-particle to a pair superfluid. The single-particle superfluid is characterized by a finite value of both the atomic condensate and the super-counterfluid density. The pair superfluid phase is found to be stable against formation of many-body cluster states and features a gap in the spectrum of elementary excitations.
\end{abstract}

DOI: 10.1103/PhysRevA.90.043623

PACS number(s): 67.85.-d, 03.75.Hh, 05.30.Jp

The study of quantum degenerate gases of dipolar particles has become in recent years one of the most active areas of experimental and theoretical research in the field of ultracold atoms $[1,2]$. The realization of systems featuring strong dipolar interactions opens prospects for investigating new and highly interesting many-body effects which arise from the anisotropic and long-range nature of the interatomic force. An example is the quest for $p$-wave superfluidity in a two-dimensional (2D) Fermi gas where dipoles are aligned by an external field at an angle formed with the plane of confinement larger than some critical value [3,4]. Another example involves fermionic dipoles in a bilayer geometry that allows for interlayer pairing of particles and displays superfluidity of pairs which, depending on the interlayer distance, ranges from a Bardeen-Cooper-Schrieffer (BCS) type to a Bose-Einstein condensate (BEC) of tightly bound dimers $[5,6]$. This latter system shares many analogies with the electron-hole bilayers realized in semiconductor coupled quantum wells $[7,8]$ as well as graphene [9], where excitonic superfluidity is predicted to occur $[10,11]$ even though a clear experimental observation is still lacking.

In this paper, we investigate two-dimensional bilayers of bosonic dipoles, where the dipoles are oriented perpendicularly to the parallel planes which provide the $2 \mathrm{D}$ confinement. Tunneling between layers is assumed to be negligible due to the high potential barrier separating the planes. If one neglects short-range forces, in-plane interactions are purely repulsive and behave as $1 / r^{3}$ in terms of the interparticle distance. On the contrary, out-of-plane interactions are attractive at short distance and might induce pairing between particles in the two layers [12].

In contrast to the fermionic counterpart, the bosonic system displays a quantum phase transition, as a function of the interlayer attraction, from a single-particle to a pair superfluid state (see Fig. 4). In the case of a tight-binding model of hardcore bosons on a lattice, the phase diagram at zero temperature has been investigated using mean field [13] and quantum Monte Carlo (QMC) methods [14] and was found to include exotic phases around half filling such as the checkerboard solid and the pair supersolid. Here we study a translationally invariant system without optical lattices in the planes and we perform simulations in the continuum by means of the diffusion Monte Carlo (DMC) technique. The ground-state energy is calculated as a function of the distance between the two layers and the emergence of off-diagonal long-range order (ODLRO) is investigated both in the one-body (OBDM) and in the two-body density matrix (TBDM). The occurrence of the quantum phase transition is signaled by the vanishing atomic condensate fraction and by the appearance of a gap in the single-particle excitation spectrum.

Also other bosonic systems on a lattice, such as twocomponent BECs, have been shown to feature pair superfluidity [15], while our study is the first to address this exotic quantum phase in continuum space using exact QMC techniques. It is worth stressing that the pair superfluid phase considered here is found to be stable against formation of cluster states. This requirement is not easily fulfilled by other proposals of Bose condensates coupled by attractive forces where particles, due to their statistics, are prone to collapse into bound states involving more than just a dimer [16].

The microscopic Hamiltonian is defined as follows:

$$
\begin{aligned}
H= & -\frac{\hbar^{2}}{2 m} \sum_{i=1}^{N / 2} \nabla_{i}^{2}-\frac{\hbar^{2}}{2 m} \sum_{\alpha=1}^{N / 2} \nabla_{\alpha}^{2} \\
& +\sum_{i<j} \frac{d^{2}}{r_{i j}^{3}}+\sum_{\alpha<\beta} \frac{d^{2}}{r_{\alpha \beta}^{3}}+\sum_{i, \alpha} \frac{d^{2}\left(r_{i \alpha}^{2}-2 h^{2}\right)}{\left(r_{i \alpha}^{2}+h^{2}\right)^{5 / 2}} .
\end{aligned}
$$

The first two terms correspond to the kinetic energy of particles of mass $m$ residing in the top layer (labeled by the index $i$ ) and in the bottom layer (labeled by the index $\alpha$ ). Each layer contains the same number $N / 2$ of particles, $N$ being the total number of dipoles. The terms in the second row of Eq. (1) correspond to the intralayer and interlayer dipolar interactions involving particles with dipole moment $d$ oriented perpendicularly to the two layers separated by a distance $h$. Here, $r_{i j(\alpha \beta)}=\left|\mathbf{r}_{i(\alpha)}-\mathbf{r}_{j(\beta)}\right|$ denotes the in-plane distance between pairs of particles in the top (bottom) layer, and $r_{\alpha i}=\left|\mathbf{r}_{\alpha}-\mathbf{r}_{i}\right|$ is the distance between the projections onto any of the layers of the positions of the $\alpha$ th and $i$ th particle.

Simulations are carried out in a 2D square box of area $A=$ $L^{2}$ with periodic boundary conditions. The number of particles used ranges from $N=60$ up to $N=180$ dipoles. The total density $n=N / A$ and the length scale $r_{0}=m d^{2} / \hbar^{2}$, arising 
from the dipole-dipole force, define the relevant dimensionless parameters of the system: the interaction strength $n r_{0}^{2}$ and the reduced distance $h / r_{0}$ between layers. An important aspect of the physics of this bilayer configuration is the existence of a bound state in the two-body problem for any value of the interlayer distance $h$ [17-19]. In the following we denote by $\epsilon_{b}$ the binding energy of the dimer which we obtain by solving numerically the Schrödinger equation $\left(-\frac{\hbar^{2}}{m} \nabla^{2}+\frac{d^{2}\left(r^{2}-2 h^{2}\right)}{\left(r^{2}+h^{2}\right)^{5 / 2}}-\right.$ $\left.\epsilon_{b}\right) \psi_{b}(\mathbf{r})=0$ for the pair wave function.

DMC simulations provide an exact result, in the statistical sense, for the energy of the ground state of the system [20]. Simulations are greatly speeded up when a physically relevant guiding wave function is used for importance sampling. Although different choices of the guiding wave function are not expected to affect the energy, nonlocal estimators might be biased. In order to confirm the independence of the results on the particular form of the guiding model, two different guiding wave functions, containing a priori different physics, have been considered. One is chosen to be of the Jastrow form,

$$
\Psi_{T}\left(\mathbf{r}_{1}, \ldots, \mathbf{r}_{N}\right)=\prod_{i<j} f_{1}\left(r_{i j}\right) \prod_{\alpha<\beta} f_{1}\left(r_{\alpha \beta}\right) \prod_{i, \alpha} f_{2}\left(r_{i \alpha}\right),
$$

where the in-plane and interlayer two-body correlation terms, $f_{1}$ and $f_{2}$, are non-negative functions of the pair relative coordinate. In-plane correlations are parametrized using for $f_{1}$ the same functional form as in Ref. [21], which accounts both for the cusp condition at short distances and for the phonon contribution at large separations. The interlayer term $f_{2}$ is taken as the solution of the two-body problem up to $r=R_{0}$, imposing the condition $f_{2}^{\prime}\left(r=R_{0}\right)=0$, where $0 \leqslant R_{0} \leqslant L / 2$ is a variational parameter to be optimized. For distances larger than $R_{0}$ we set $f_{2}(r)=1$. As a second choice we have adopted an alternative guiding function which explicitly accounts for the formation of pairs [22],

$$
\begin{aligned}
\Psi_{T}^{\mathrm{pair}}\left(\mathbf{r}_{1}, \ldots, \mathbf{r}_{N}\right)= & \prod_{i<j} f_{1}\left(r_{i j}\right) \prod_{\alpha<\beta} f_{1}\left(r_{\alpha \beta}\right) \\
& \times\left(\prod_{i=1}^{N / 2} \sum_{\alpha=1}^{N / 2} \tilde{f}_{2}\left(r_{i \alpha}\right)+\prod_{\alpha=1}^{N / 2} \sum_{i=1}^{N / 2} \tilde{f}_{2}\left(r_{i \alpha}\right)\right) .
\end{aligned}
$$

In-plane correlations are described in the same way by $\Psi_{T}$ and $\Psi_{T}^{\text {pair }}$, while in (3) interlayer ones are rearranged in such a way that (though preserving Bose symmetry in each layer) emergence of a given pairing between particles in the bottom and top layer is favored. This is achieved setting $\tilde{f}_{2}(r)=e^{-a r^{2} /(1+b r)}$ and using $a$ and $b$ as variational parameters which we optimize.

The equation of state as a function of the interlayer distance $h / r_{0}$ at the density $n r_{0}^{2}=1$ is shown in Fig. 1 . The red solid and blue open symbols correspond to the results obtained using guiding wave functions (2) and (3) extrapolated to the thermodynamic limit. The inset in Fig. 1 displays the energy per particle $E / N$ compared to half of the dimer binding energy $\epsilon_{b} / 2$, while in the main figure we show the variation of the difference $E / N-\epsilon_{b} / 2$ with the distance $h / r_{0}$. We notice that $E / N$ becomes negative when the interlayer distance gets small enough and approaches the dimer binding energy in the limit

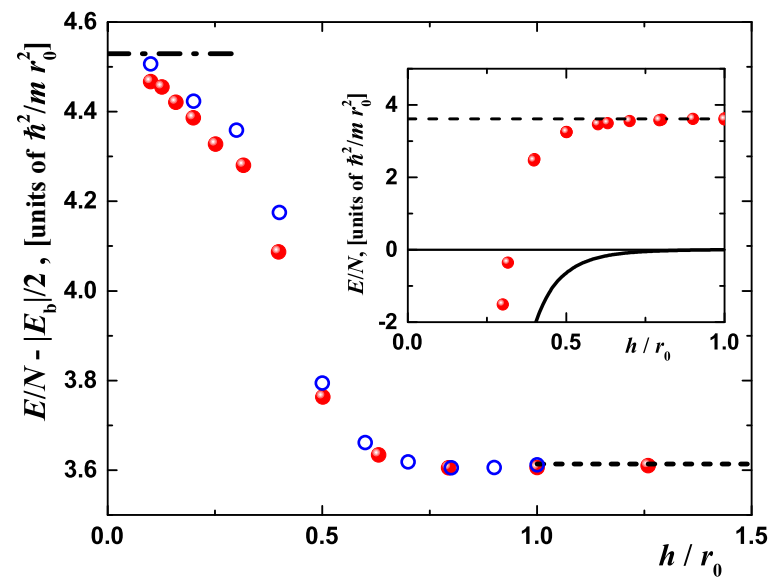

FIG. 1. (Color online) Energy per particle with half of the dimer binding energy subtracted as a function of the reduced interlayer distance $h / r_{0}$ for $n r_{0}^{2}=1$. Red solid and blue open symbols correspond to the results obtained using the guiding wave functions in Eqs. (2) and (3), respectively. The horizontal lines correspond to the energies of a single layer of dipoles with effective interaction strength $\tilde{n} \tilde{r}_{0}^{2}=0.5$ and $\tilde{n} \tilde{r}_{0}^{2}=32$. (Inset) Energy per particle $\left(n r_{0}^{2}=1\right)$ and $\epsilon_{b} / 2$ (solid line) as a function of the interlayer separation. In both cases the dashed line corresponds to the large $h / r_{0}$ limit.

$h \ll r_{0}$. An important remark is that in this regime the energy difference $E / N-\epsilon_{b} / 2$ is found to be positive, indicating that dimers feel an effective repulsive interaction which stabilizes the pair phase.

Pairing between dipoles is in fact a strong effect when $h \ll r_{0}$, forming tightly bound dimers which behave as composite objects featuring twice the mass and dipole moment as compared to single dipoles. The horizontal lines in Fig. 1 correspond to the energies per particle of a single layer of dipolar bosons with an effective interaction strength $\tilde{n} \tilde{r}_{0}^{2}$, as obtained using the results of Refs. [21,23], where $\tilde{n}=n / 2$ and the dipolar length takes the two values $\tilde{r}_{0}=r_{0}$ and $\tilde{r}_{0}=8 r_{0}$. The first value corresponds to the asymptotic regime $h \gg r_{0}$ of independent layers, whereas the second value refers to the opposite regime, $h \ll r_{0}$, where the system behaves as a single layer of particles having dipole moment $2 d$ and mass $2 m$ as mentioned above.

After discussing the equation of state we analyze the OBDM and TBDM as a function of the interlayer distance and the nature of the transition between single-particle and pair superfluidity. The OBDM within each layer is defined as

$$
\rho_{1}(s)=\frac{2}{n}\left\langle\psi_{t(b)}^{\dagger}(\mathbf{r}+\mathbf{s}) \psi_{t(b)}(\mathbf{r})\right\rangle
$$

where $\psi_{t(b)}^{\dagger}(\mathbf{r}), \psi_{t(b)}(\mathbf{r})$ correspond to the creation/annihilation operators of a particle at the 2D coordinate $\mathbf{r}$ in the top (bottom) layer. The relevant TBDM involves instead a pair of particles residing in different layers [24],

$$
\rho_{2}(s)=\frac{2}{n} \int d \mathbf{r}^{\prime}\left\langle\psi_{b}^{\dagger}(\mathbf{r}+\mathbf{s}) \psi_{t}^{\dagger}\left(\mathbf{r}^{\prime}+\mathbf{s}\right) \psi_{t}\left(\mathbf{r}^{\prime}\right) \psi_{b}(\mathbf{r})\right\rangle .
$$

Notice the different normalization of the two functions at $s=$ $0: \rho_{1}(0)=1$ and $\rho_{2}(0)=N / 2$. 


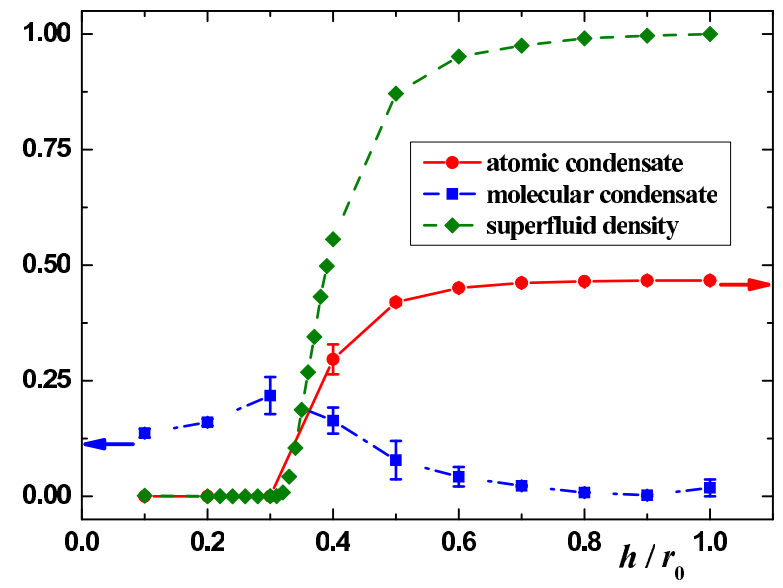

FIG. 2. (Color online) Atomic condensate $n_{0}$ and molecular condensate $n_{0}^{\text {mol }}$ as a function of $h / r_{0}$ at the density $n r_{0}^{2}=1$. Arrows correspond to the condensate fraction of a single layer of dipoles at the effective interaction strength $\tilde{n} \tilde{r}_{0}^{2}=0.5$ (red arrow) and $\tilde{n} \tilde{r}_{0}^{2}=32$ (blue arrow). The dashed green line and symbols correspond to the super-counterfluid density of Eq. (6).

For homogeneous systems, ODLRO in the OBDM implies a finite value of Eq. (4) at large separations: $\lim _{s \rightarrow \infty} \rho_{1}(s)=n_{0}$, where $n_{0} \leqslant 1$ is the fraction of atoms in the condensate of each layer. Similarly, ODLRO in the TBDM entails that $\lim _{s \rightarrow 0} \rho_{2}(s)=\alpha$. One should notice that ODLRO at the level of the OBDM implies ODLRO also at the level of the TBDM and, in this case, $\alpha=(N / 2) n_{0}^{2}$, which is macroscopically large. However, even if ODLRO is absent in the OBDM $\left(n_{0}=0\right)$, it can still be present in the TBDM, and $\alpha(<1)$ is interpreted as the condensate fraction of pairs [25]. An intrinsic order parameter related to the TBDM can be defined as [26]: $\lim _{s \rightarrow \infty} \rho_{2}(s)-(N / 2) n_{0}^{2}=n_{0}^{\mathrm{mol}}$. The molecular condensate fraction $n_{0}^{\text {mol }}$ coincides with the long-range behavior $\alpha$ of the TBDM when the atomic condensate $n_{0}$ vanishes and one removes from it the largest contribution, which scales as the total number of particles, when $n_{0} \neq 0$.

The calculation of the OBDM and TBDM using the DMC method relies on an extrapolation technique based on both DMC and variational Monte Carlo (VMC) results in order to extract the expectation value of the relevant operator on the ground state of the system [20]. The estimate obtained in this way is unbiased only if the guiding wave function $\psi_{T}$ used in the VMC calculation, and as importance sampling in the DMC simulation, do not differ too much from the true ground-state wave function. In order to make sure that our results for the density matrices do not depend on the special choice of the guiding wave function (2), we carried out DMC simulations starting from the two wave functions in Eqs. (2) and (3).

Results for the OBDM and TBDM are shown in Fig. 2 for the density $n r_{0}^{2}=1$. For both the particle and the molecular condensate, an average between the determination using guiding functions (2) and (3) has been performed, and the error bars reported in the figures give an indication of how close the two estimates are. The particle condensate is clearly vanishing for interlayer distances smaller than a critical value, and grows continuously until it reaches the value corresponding to a single layer of dipoles at the density $n r_{0}^{2} / 2$ [21]. The molecular condensate fraction $n_{0}^{\text {mol }}$ is extremely small in the regime of weak pairing corresponding to large interlayer separations, and increases smoothly in the region of the transition to the molecular regime until it reaches the value expected for a single layer of dipolar dimers. We notice that for values of $h$ in the range $0.3 \lesssim h / r_{0} \lesssim 0.6$ both $n_{0}$ and $n_{0}^{\text {mol }}$ are appreciably different from zero.

In Fig. 2 we also show the results of the super-counterfluid density defined as [14]

$$
\rho_{s}=\lim _{\tau \rightarrow \infty} \frac{\left\langle\left(\mathbf{W}_{t}(\tau)-\mathbf{W}_{b}(\tau)\right)^{2}\right\rangle}{2 N \tau},
$$

and written in terms of the winding number relative to the top and bottom layer,

$$
\mathbf{W}_{t(b)}(\tau)=\sum_{i(\alpha)=1}^{N / 2} \int_{0}^{\tau} d \tau^{\prime}\left(\frac{d \mathbf{r}_{i(\alpha)}\left(\tau^{\prime}\right)}{d \tau^{\prime}}\right),
$$

where $\mathbf{r}_{i(\alpha)}$ is the in-plane coordinate of the particles belonging to the top (bottom) layer. We notice that when the imaginarytime evolution of the particles in the top and bottom layer is fully correlated, as happens in the paired phase, the contribution to $\rho_{s}$ vanishes, while $\rho_{s}=1$ when the two layers behave as independent superfluids. The results for $\rho_{s}$ and $n_{0}$ in Fig. 2 show that both quantities vanish at the same critical interlayer distance where the system enters the pair superfluid phase. This behavior is consistent with a second-order phase transition, similarly to that reported in Ref. [14] for the case of a bilayer system of dipolar bosons in an optical lattice.

The phase transition from the atomic to the pair superfluid is further characterized by the appearance of a gap in the spectrum of elementary excitations. The gap value can be calculated from the dependence of the ground-state energy $E(P)$ on a small polarization $P=\left(N_{t}-N_{b}\right) / N(|P| \ll 1)$ obtained by slightly unbalancing the populations $N_{t(b)}$ of the top (bottom) layer while keeping the total number $N=$ $N_{t}+N_{b}$ fixed. If an atomic condensate is present $\left(n_{0} \neq 0\right)$, the low-lying excitations are coupled phonon modes of the two layers. In this case $E(P)=E(0)+N\left(n / 2 \chi_{s}\right) P^{2}$, where $E(0)$ is the ground-state energy of the balanced system and $\chi_{s}$ is the spin susceptibility associated with the dispersion of spin waves of the magnetization density $n_{t}-n_{d}$ with speed of sound $c_{s}=\sqrt{n / m \chi_{s}}$. In the pair superfluid phase an energy $\Delta$ is needed to break a pair and spin excitations are gaped. The resulting energy $E(P)=E(0)+N \Delta P$ is linear in the polarization. Examples of the different behavior of $E(P)$ is shown in the inset of Fig. 3 for two values of the interlayer distance corresponding to the atomic and pair superfluid. The values of the gap $\Delta$ extracted from the fits to $E(P)$ are shown in Fig. 3. The gap grows continuously from zero starting from a critical interlayer distance $h_{c}$ and for smaller separations it remains close to the value $\epsilon_{b} / 2$ set by the dimer binding energy. The estimated value $h_{c} \simeq 0.35 r_{0}$ for the critical distance is consistent with the results of the condensate and super-counterfluid density of Fig. 2.

Figures 1-3 report results obtained for the gas parameter $n r_{0}^{2}=1$. Qualitatively similar results are found for different values of $n r_{0}^{2}$. A schematic phase diagram is shown in Fig. 4, where the estimated critical interlayer distance $h_{c}$ is reported for three different densities. The figure also shows the freezing 


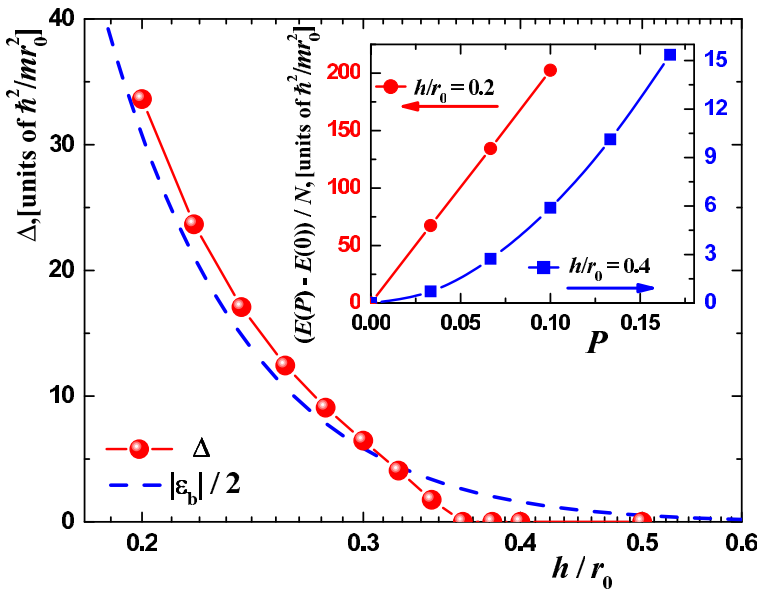

FIG. 3. (Color online) Excitation gap $\Delta$ as a function of $h / r_{0}$ at the density $n r_{0}^{2}=1$. (Red symbols and line) DMC results and guide to the eye. The dashed blue line corresponds to $\epsilon_{b} / 2$. (Inset) Energy $E(P)$ for two values of $h / r_{0}$ in the pair and single-particle superfluid phase with the corresponding linear and quadratic fit.

density where the triangular solid is formed, occurring at $\tilde{n} \tilde{r}_{0}^{2} \simeq$ 290 [21,27], for the single layer of particles $\left(\tilde{r}_{0}=r_{0}\right)$ and of pairs $\left(\tilde{r}_{0}=8 r_{0}\right)$.

In summary, in this work we have analyzed the behavior of a bilayer system of perpendicularly aligned dipolar bosons in the continuum. While the two-body problem always has a bound state, we have shown that the many-body system undergoes a phase transition from a molecular phase of tightly bound pairs to a single-particle phase as the distance between the layers increases. Our simulations show that the phase transition is characterized by the atomic condensate fraction and the super-counterfluid density which decay to zero simultaneously when the interlayer distance is reduced below a certain critical value that depends on the density. At the same critical value, a gap in the excitation spectrum opens and the system enters the pair superfluid phase. We also notice that

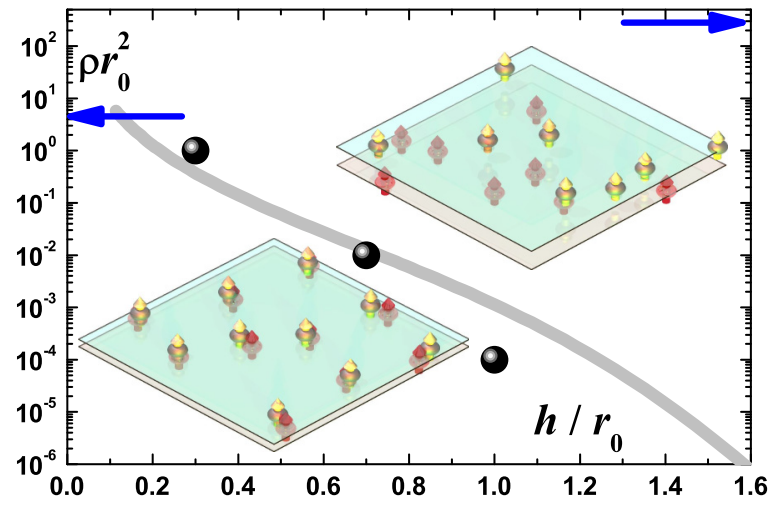

FIG. 4. (Color online) Schematic phase diagram featuring the single-particle (upper region) and the pair superfluid (lower region). The dots correspond to the transition points as obtained from DMC simulations. The two arrows show the freezing density of a single layer of particles (right) and of dimers (left). The line separates the region where $\left|\epsilon_{b}\right| / 2<\mu$ (weak pairing) from the region where $\left|\epsilon_{b}\right| / 2>\mu$ (strong pairing).

the single-particle superfluid phase has the peculiar feature of exhibiting ODLRO both in the OBDM and in the TBDM with an intrinsic character. We are not aware of any other superfluid state showing such a property. Remarkably, a bilayer system of dipoles is, to the best of our knowledge, the first example of a physical system exhibiting bosonic pair superfluidity in the continuum that could be explored in current experiments on ultracold gases with predominant dipolar interactions.

We acknowledge partial financial support from DGI (Spain) Grant No. FIS2011-25275 and Generalitat de Catalunya Grant No. 2009SGR-1003. G.E.A. acknowledges a fellowship from MEC (Spain) through the Ramon y Cajal program. S.G. acknowledges support from ERC through the QGBE grant and from Provincia Autonoma di Trento. Part of the numerical simulations were carried out at the Barcelona Supercomputing Center (the Spanish National Supercomputing Center-Centro Nacional de Supercomputación).
[1] M. A. Baranov, Phys. Rep. 464, 71 (2008).

[2] M. A. Baranov, M. Dalmonte, G. Pupillo, and P. Zoller, Chem. Rev. 112, 5012 (2012).

[3] G. M. Bruun and E. Taylor, Phys. Rev. Lett. 101, 245301 (2008).

[4] L. M. Sieberer and M. A. Baranov, Phys. Rev. A 84, 063633 (2011).

[5] A. Pikovski, M. Klawunn, G. V. Shlyapnikov, and L. Santos, Phys. Rev. Lett. 105, 215302 (2010).

[6] N. Matveeva and S. Giorgini, arXiv:1405.7588.

[7] U. Sivan, P. M. Solomon, and H. Shtrikman, Phys. Rev. Lett. 68, 1196 (1992).

[8] J. A. Seamons, C. P. Morath, J. L. Reno, and M. P. Lilly, Phys. Rev. Lett. 102, 026804 (2009).

[9] R. V. Gorbachev, A. K. Geim, M. I. Katsnelson, K. S. Novoselov, T. Tudorovskiy, I. V. Grigorieva, A. H. MacDonald, S. V. Morozov, K. Watanabe, T. Taniguchi, and L. A. Ponomarenko, Nat. Phys. 8, 896 (2012).
[10] Yu. E. Lozovik and V. I. Yudson, Pis'ma Zh. Eksp. Teor. Fiz. 22, 556 (1975) [JETP Lett. 22, 274 (1975)]; Solid State Commun. 19, 391 (1976); Zh. Eksp. Teor. Fiz. 71, 738 (1976) [Sov. Phys. JETP 44, 389 (1976)].

[11] A. Perali, D. Neilson, and A. R. Hamilton, Phys. Rev. Lett. 110, 146803 (2013).

[12] Dai-Wei Wang, Phys. Rev. Lett. 98, 060403 (2007).

[13] C. Trefzger, C. Menotti, and M. Lewenstein, Phys. Rev. Lett. 103, 035304 (2009).

[14] A. Safavi-Naini, S. G. Soyler, G. Pupillo, H. R. Sadeghpour, and B. Capogrosso-Sansone, New J. Phys. 15, 013036 (2013).

[15] A. Kuklov, N. Prokof'ev, and B. Svistunov, Phys. Rev. Lett. 92, 030403 (2004).

[16] P. Nozières and D. Saint James, J. Phys. 43, 1133 (1982).

[17] B. Simon, Ann. Phys. 97, 279 (1976).

[18] J. R. Armstong, N. T. Zinner, D. V. Fedorov, and A. S. Jensen, EuroPhys. Lett. 91, 16001 (2010). 
[19] M. Klawunn, A. Pikovski, and L. Santos, Phys. Rev. A 82, 044701 (2010).

[20] See, e.g., J. Kolorenč and L. Mitas, Rep. Prog. Phys. 74, 026502 (2011).

[21] G. E. Astrakharchik, J. Boronat, I. L. Kurbakov, and Yu. E. Lozovik, Phys. Rev. Lett. 98, 060405 (2007).

[22] C. Cazorla, G. E. Astrakharchik, J. Casulleras, and J. Boronat, New J. Phys. 11, 013047 (2009).
[23] G. E. Astrakharchik, J. Boronat, J. Casulleras, I. L. Kurbakov, and Yu. E. Lozovik, Phys. Rev. A 75, 063630 (2007).

[24] G. E. Astrakharchik, J. Boronat, J. Casulleras, and S. Giorgini, Phys. Rev. Lett. 95, 230405 (2005).

[25] C. N. Yang, Rev. Mod. Phys. 34, 694 (1962).

[26] D. H. Kobe, J. Math. Phys. 10, 1507 (1969).

[27] H. P. Büchler, E. Demler, M. Lukin, A. Micheli, N. Prokof'ev, G. Pupillo, and P. Zoller, Phys. Rev. Lett. 98, 060404 (2007). 